\title{
Cerebral Schistosomiasis - An Unusual Presentation of an Intracranial Mass Lesion
}

\author{
Jason George, David Rose, Lili-Naz Hazrati, Lance Majl, Mojgan Hodaie
}

Can. J. Neurol. Sci. 2009; 36: 244-247

Schistosomiasis is an intravascular trematode infection affecting at least 200 million people worldwide ${ }^{1}$. Trematodes are parasitic worms that are flattened dorsoventrally. Their suckers (trematos, Greek 'holes'), act as organs of attachment. Schistosome worms are blood flukes with a complex life cycle involving freshwater snails that act as intermediate hosts, and the bloodstream of higher order vertebrates that are the definitive hosts. The three major schistosome species ( $S$. mansoni, $S$. japonicum and $S$. haematobium) known to infect humans usually reside in the venous systems of the liver, intestines or urinary bladder. Schistosomal species have different geographical distributions. Schistosoma haematobium is encountered mainly in Africa and the Middle East; S. mansoni is additionally seen in South America and the Caribbean; and $S$. japonicum is primarily found in the Far East ${ }^{2}$. The main clinical features are related to the life cycle of the schistosome. Initially, larvae from freshwater penetrate the skin resulting in a cercarial dermatitis or 'swimmer's itch'. Subsequently, a systemic hypersensitivity response may occur, known as Katayama fever, in response to the migrating schistosomulae ${ }^{3}$. Finally, chronic pathology is due to granuloma formation in response to egg deposition ${ }^{4}$.

Involvement of the central nervous system (CNS) is uncommon but can be an important complication. The risk of CNS infection depends on the level of immunity and the magnitude of the schistosomal invasion. Histologically, the changes seen in the CNS correspond to the host's inflammatory reactions to the ova and range from nothing to florid granulomatous inflammation. There are two theories as to how the eggs reach the CNS. Eggs from the portal system could embolise to the brain along the vertebral venous (Batson) plexus $^{5}$, via atrial/septal defects or patent foramen ovale, or via pulmonary venous shunts, as a result of hepatic and pulmonary hypertension ${ }^{6}$. Alternatively, the adult worms could enter the Batson plexus and then migrate upwards to the brain and deposit directly their eggs ${ }^{7}$. The small size and round shape of $\mathrm{S}$. japonicum ova favor an easier migration to the brain, according to the embolisation hypothesis. Conversely, S. mansoni and haematobium ova are larger, with $S$. mansoni possessing a prominent lateral spine. These differences may explain the relatively more common presentation of transverse myelitis and other spinal syndromes when neurological complications ensue with these species ${ }^{8}$. Once in the brain, soluble egg antigens are responsible for provoking a peri-ova granulomatous reaction, comprising lymphocytes, macrophages and eosinophils. Host factors determine the severity of response ${ }^{4}$.
We describe a case of cerebral schistosomiasis presenting as a newly onset seizure and a multifocal mass-like lesion in the brain.

\section{Case Report}

A 23-year-old right-handed woman of Filipino origin, presented to our hospital with a recent history of focal seizures. Her first recorded seizure had occurred six months earlier, when she was in the Philippines. This was a single event and did not recur until she arrived in Canada, approximately six months later. The attacks were stereotyped in nature with right hemisensory disturbance followed by sequential tonic contraction of the right arm and right hemifacial tonic spasm associated with transient motor aphasia. The seizures were preceded by numbness on the right side. Shortly after arriving to Canada and just prior to presenting to the hospital, she experienced an increase in the frequency of the seizures, reporting about a dozen of attacks in a short period of time. She denied having any headaches, visual disturbance or weight loss. Anticonvulsant treatment with carbamazepine was commenced with adequate seizure control.

Her past medical history was significant for rheumatic fever as a child but no chronic cardiac sequelae as shown by a normal echocardiogram. She was brought up on a farm in a small village on the island of Mindanao, in the southern Philippines; this involved contact with many animals including dogs, cows and sheep. She also had exposure to freshwater. There was no previous history of neurological, gastrointestinal or genitourinary symptoms, nor did she have fever or night sweats. There was a strong family history of tuberculosis (TB), including a grandmother who had lived with the patient and died from the disease.

On examination, she appeared fully awake, alert and oriented.

\footnotetext{
From Imperial College (JG), London, United Kingdom; Division of Infectious diseases (DR), Division of Neurology (LM), The Scarborough Hospital; Department of Pathology (LH), Division of Neurosurgery (MH), University Health Network and University of Toronto, Toronto, Ontario.

Received April 4, 2008. Final Revisions Submitted November 12, 2008. Correspondence to: Mojgan Hodaie, Toronto Western Hospital, 4WW-443, 399 Bathurst St., Toronto, Ontario, M5T 2S8, Canada.
} 


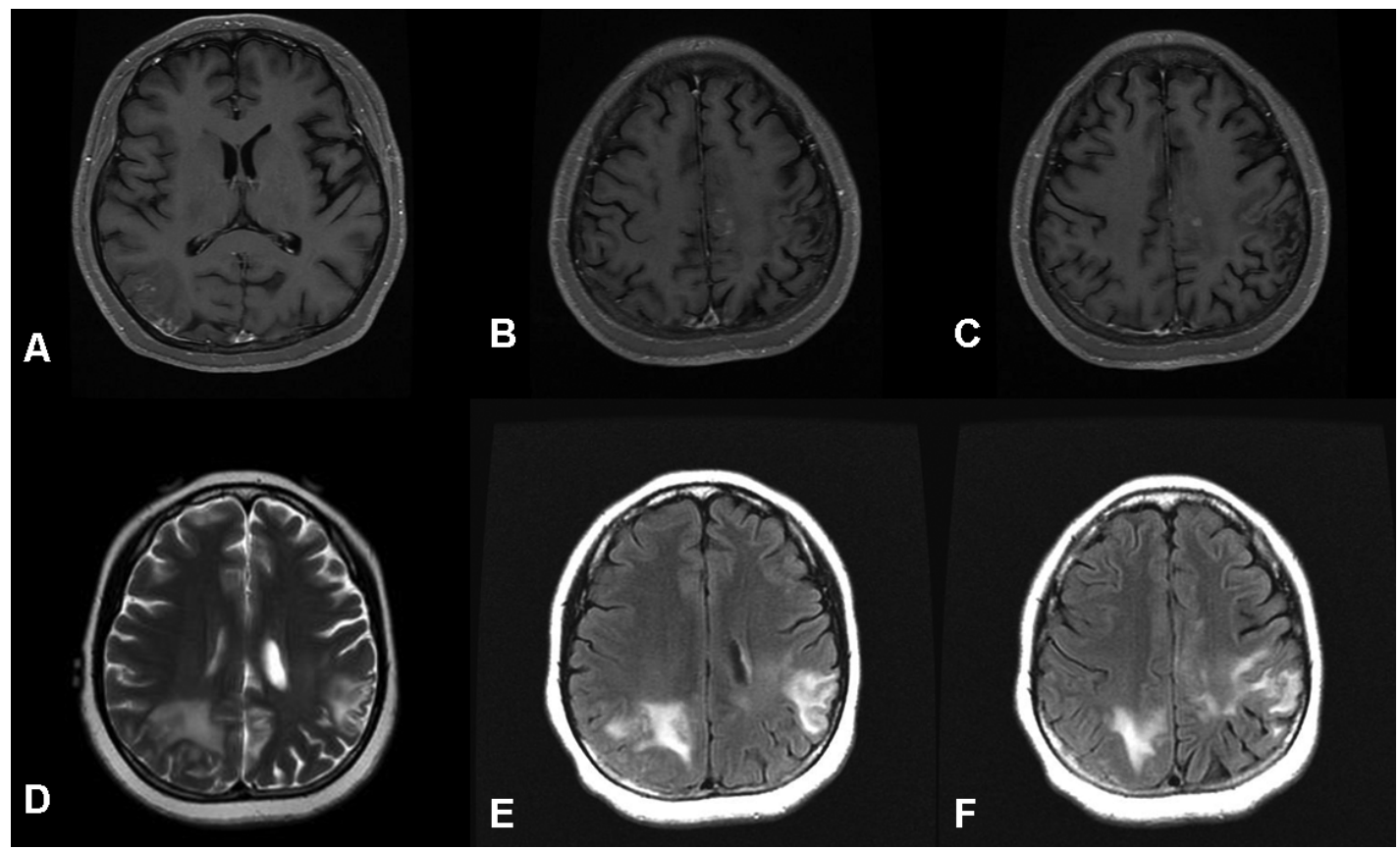

Figure 1: Magnetic resonance imaging of the brain. Axial T1 with gadolinium enhancement shows enhancing of the cortex and gyri in the right parietal area, with no evidence of diffuse leptomeningeal disease (1A). Mild left parasagittal enhancement can also be visualized $(1 B, 1 C)$ together with evidence of left parietal gliosis. T2 axial (1D) and FLAIR images $(1 E, 1 F)$ reveal the degree of bilateral edema surrounding the lesions, out of keeping with the size of the areas of enhancement.

Her vital signs were normal and general physical examination was unremarkable. In particular, there was no hepatosplenomegaly or lymphadenopathy. Her extraocular movements were full with no nystagmus. Visual fields were full on confrontation. Fundoscopy did not reveal any papilloedema. Tone, power and reflexes were all within the normal range and there was no sensory loss.

Multiple imaging studies were carried out. Computed tomogram (CT) scan revealed partially calcified lesions with significant uptake of CT contrast dye. The lesions were serpentine in appearance and located primarily in the left frontoparietal and right parieto-occipital lobes. Significant vasogenic edema was seen to be associated with the lesions (Figure 1). Magnetic resonance imaging (MRI) was done with and without gadolinium enhancement and showed multifocal lesions associated with significant edema and mass effect. The lesions appeared to involve gray and white matter however leptomeningeal involvement could not be distinguished. Compared to the contrast uptake seen with CT imaging, little enhancement of the lesions was seen with gadolinium. Magnetic resonance angiography did not demonstrate any vascular lesions. Complete blood work, electrolytes and cerebrospinal fluid (CSF) analysis were within normal range. The early differential diagnosis included multifocal neoplastic or inflammatory processes as well as infectious processes such as cerebral tuberculosis.

Given her strong family history of TB exposure and despite normal chest radiography, we favored cerebral TB as our main differential diagnosis. She was started on a drug regimen for probable cerebral TB (Isoniazid, rifampin, pyrazinamide, ethambutol and vitamin B6). Clinically, the patient remained afebrile, was compliant with anticonvulsant medication and free from seizures. Her main symptoms consisted of fleeting headaches and occasional transient paresthesia in the right arm.

Follow-up imaging revealed interval worsening of some of the lesions and improvement of others. An infectious/ granulomatous lesion was then favored and biopsy and tissue confirmation of the diagnosis recommended. The patient underwent a right parietal craniotomy and frameless stereotactic biopsy. Upon opening the dura, the pia and brain surface appeared clearly abnormal, with evidence of an inflammatory reaction that involved the leptomeninges and the dura. Frozen section examination of the biopsy specimen revealed presence of granuloma. Microscopic examination revealed clusters of multinucleate giant cells, monocytes, lymphocytes and eosinophils. The granulomatous reaction involved the dura, the leptomeninges, the brain parenchyma as well as Virchow-Robin spaces. The surrounding and adjacent brain tissue showed reactive gliosis. At the centre of these granulomata were multiple schistosomal eggs with a distinctive lateral spine. (Figure $2 \mathrm{~A}-\mathrm{D}$ ) The staining pattern and morphology were characteristic of both $S$. mansoni and S. japonicum species. However, given the patient's epidemiological history of emigrating from the Philippines, a diagnosis of S.japonicum infection was preferred.

Postoperatively the patient was treated with praziquantel $(20 \mathrm{mg} / \mathrm{kg}$ tid x 1 day) and dexamethasone, on a tapered dose for 


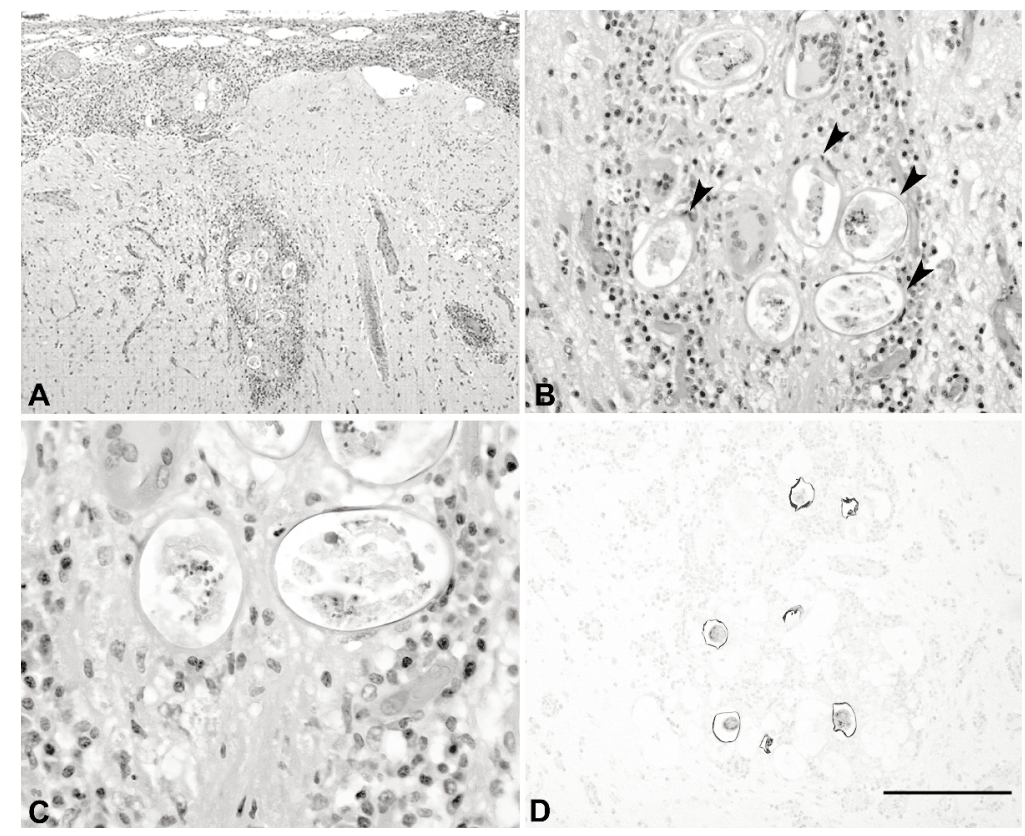

Figure 2: Microphotographs of the pathological findings in the brain biopsy. A: An intense granulomatous inflammatory reaction to the Schistosoma ova involves the leptomeninges, the Virchow-Robin spaces and the brain parenchyma $(A: 5 X) ; B-C$ : High power details of the Schistosoma ova (B:20X;C:40X). The cuticle of the ova stains strongly with Ziehl-Nelson (D:10X).

three weeks. The patient has remained on carbamazepine with no further seizures. Repeat MRI at four months showed decrease in cerebral edema and FLAIR signal but some evidence of gliosis. Her neurological symptoms have resolved completely at follow-up.

\section{Discussion}

Yamagiwa, a student of Rudolf Virchow, published the first case of cerebral schistosomiasis due to $S$. japonicum in $1889^{9}$. However, S. japonicum first achieved global interest during World War II when American forces gathered on Leyte Island in the Philippines, as part of the Pacific campaign. Troops wading through freshwater swamps and streams, heavily populated with the Oncomelania snail intermediate host, went on to develop the disease. Out of 1200 soldiers with acute $S$. japonicum infection, $2.3 \%$ developed cerebral complications ${ }^{10}$.

Cerebral schistosomiasis due to japonicum species may present acutely as a diffuse encephalopathy with features including delirium, focal motor deficits, visual and speech disturbances - usually in association with Katayama syndrome ${ }^{11}$. More commonly, intracranial granulomas may develop and present as mass lesions, as seen in our case, sometimes many years after initial infection. These granulomas often provoke focal or generalized seizures, reminiscent of the earliest case description (including visualization of $S$. japonicum ova) by Yamagiwa. The CT typically reveals an enhancing mass lesion associated with edema and without calcification. An MRI may show a heterogeneously enhancing mass with surrounding edema $^{12,13}$. Such brain lesions are frequently asymptomatic when found in the more severe, chronic forms of S. haematobium and S. mansoni infection ${ }^{8}$. However large granulomatous lesions provoking brain tumor-like syndromes have been described in occasional S. mansoni cases, giving rise to increased intracranial pressure and focal neurological signs ${ }^{14}$. A second pattern can be apparent on MR, primarily consisting of a nodular and linear enhancement pattern with associated vasogenic edema. In these instances, the pattern of contrast enhancement is more noteworthy than the associated vasogenic edema ${ }^{15}$. This indicates that the MR features of cerebral schistosomiasis can be quite indistinct, possibly presenting only with linear or nodular enhancement, rather than a mass lesion. Given the possible varied patterns seen on neuroradiological examinations and the rarity of this entity, the gold standard still remains diagnosis based on a biopsy specimen.

Raised total protein levels, pleocytosis and eosinophilia (suggestive of parasitic infection) can sometimes be observed in the CSF. Blood tests may also show eosinophilia ${ }^{5}$, however peripheral eosinophilia is uncommon in chronic infections, as seen also in our case. Kato-Katz analysis of stool samples may be positive for eggs although patients with neurological complications are often less likely to show evidence of systemic disease ${ }^{16}$. Furthermore, in patients from endemic countries recovery of eggs from urine or feces is non-diagnostic of neurological disease. Similarly, a positive serological test will 
not distinguish active infection from past exposure to the parasite. Hepatic ultrasound may show the presence of Symmer's pipestem fibrosis, a characteristic portal vein fibrosis thought to be induced by the presence of large numbers of schistosomal eggs in hepatic tissue ${ }^{17}$. Definitive diagnosis is only possible after biopsy, revealing granulomata composed of giant cells, lymphocytes and eosinophils, centered around schistosoma eggs. Serological studies (FAST-ELISA) for antibodies to the schistosomes can be considered for subtyping of the schistosomal species.

Our patient had no history of 'cercarial dermatitis', possibly due to light exposure to infested freshwaters or perhaps because it went unrecognized. She also denied any history of systemic features suggestive of Katayama syndrome and reported no gastrointestinal symptoms, normally seen with $S$. japonicum infection. These negative findings could be explained by the 'in utero sensitization' hypothesis - for individuals from chronically exposed populations ${ }^{18,19}$. According to this theory, sensitization occurs in utero as a result of maternal infection and therefore any subsequent schistosome infection in later life provokes a diminished pro-inflammatory response resulting in less severe morbidity. Normal examination and laboratory findings (on blood and CSF analysis) added to the diagnostic difficulty. Computed tomogram scanning suggested that calcifications, probably vascular in nature, were present in the mass lesions. Our differential diagnosis included primary neoplastic processes including oligodendroglioma, vascular lesions such as arteriovenous malformations, granulomatous angiitis, neurosarcoidosis and infectious processes such as cerebral tuberculosis and cysticercosis. With the benefit of histological diagnosis we may postulate that the serpiginous calcifications seen on $\mathrm{CT}$ represent focal zones of intense tissue reaction to eggs, leading to chronic pathology involving the vasculature.

There appear to be key epidemiological differences between $S$. japonicum infections and other schistosomal species. $S$. japonicum have multiple mammalian vertebrate hosts compared to only few hosts for other species. Wild and domestic animals such as dogs, cats, cattle, sheep, goats, pigs, water buffaloes and others make control of japonicum infections particularly difficult. Furthermore, snails serving as intermediate hosts for $S$. japonicum can live both in and out of water. This results in water treatments being ineffective. S. japonicum also produces more eggs compared with other schistosomal species. These eggs are smaller and have a round shape, which aids their distal embolisation in the circulation.

Apart from descriptions of American soldiers returning from the Pacific campaign of WWII ${ }^{10,20}$, we are not aware of any further reports of cerebral schistosomiasis due to $S$. japonicum presenting in North America. In contrast, there are several recent cases in the literature of S. mansoni and haematobium infection presenting with neurological complications outside their endemic regions ${ }^{12-14,21,22}$. Although focal onset seizures are the most common mode of presentation with $S$. japonicum, the diagnosis may be missed by clinicians assessing these patients residing in non-endemic areas. Awareness of geographical distributions of disease ( $S$. japonicum in China, Indonesia and the Philippines) would reduce the possibility of diagnostic error.

The treatment of cerebral schistosomiasis is highly effective and safe. Praziquantel kills the adult worms and concomitant corticosteroids reduce the granulomatous inflammation ${ }^{22}$ and are used for all schistosomal subtypes. The ease of global travel and population migration today means that it is important to note cerebral schistosomiasis due to japonicum species in the differential of unexplained seizures and non-specific imaging changes, when there is a history of exposure in an endemic region. Making the diagnosis is challenging, as manifestations may be protean and presentation with neurological complications can occur several years after the initial infection. Therefore, we recommend a high index of suspicion and an aggressive diagnostic approach in comparable cases. Early diagnosis and treatment can minimize the resultant tissue damage and eliminate the parasite worm.

\section{REFERENCES}

1. Chitsulo L, Engels D, Montresor A, Savioli L. The global status of schistosomiasis and its control. Acta Trop. 2000;77(1):41-51.

2. Blanchard TJ. Schistosomiasis. Travel Med Infect Dis. 2004;2(1):5-11.

3. Ross AG, Vickers D, Olds GR, Shah SM, McManus DP. Katayama syndrome. Lancet Infect Dis. 2007;7(3):218-24.

4. Gryseels B, Polman K, Clerinx J, Kestens L. Human schistosomiasis. Lancet. 2006;368(9541):1106-18.

5. Liu LX. Spinal and cerebral schistosomiasis. Semin Neurol. 1993; 13(2):189-200

6. Pittella JE, Gusmao SN, Carvalho GT, da Silveira RL, Campos GF. Tumoral form of cerebral Schistosomiasis mansoni. A report of four cases and a review of the literature. Clin Neurol Neurosurg. 1996;98(1):15-20.

7. Carod-Artal FJ, Vargas AP, Horan TA, Marinho PB, Coelho Costa PH. Schistosoma mansoni myelopathy: clinical and pathologic findings. Neurology. 2004;63(2):388-91.

8. Scrimgeour EM, Gajdusek DC. Involvement of the central nervous system in Schistosoma mansoni and S. haematobium infection. A review. Brain. 1985; 108(4):1023-38.

9. Yamagiwa K. Beitrage zur aetiologie der jacksonchen epilepsie. Virchows. Arch Pathol Anat. 1889;119:449-60.

10. Kane C, Most H. Schistosomiasis of the central nervous system. Arch Neurol Psychiatry. 1948;59:141-83.

11. Jaureguiberry S, Ansart S, Perez L, Danis M, Bricaire F, Caumes E. Acute neuroschistosomiasis: two cases associated with cerebral vasculitis. Am J Trop Med Hyg. 2007;76(5):964-6.

12. Preidler KW, Riepl T, Szolar D, Ranner G. Cerebral schistosomiasis: MR and CT appearance. AJNR Am J Neuroradiol. 1996;17(8):1598-600.

13. Mehta A, Teoh SK, Schaefer PW, Chew FS. Cerebral schistosomiasis. AJR Am J Roentgenol. 1997;168(5):1322.

14. Mackenzie IR, Guha A. Manson's schistosomiasis presenting as a brain tumor. Case report. J Neurosurg. 1998;89(6):1052-4.

15. Sanelli PC, Lev MH, Gonzalez RG, Schaefer PW. Unique linear and nodular MR enhancement pattern in schistosomiasis of the central nervous system: report of three patients. AJR Am J Roentgenol. 2001;177(6)1471-4.

16. Carod-Artal FJ. Neurological complications of Schistosoma infection. Trans R Soc Trop Med Hyg. 2007;102(2):107-116.

17. Richter J, Hatz C, Haussinger D. Ultrasound in tropical and parasitic diseases. Lancet. 2003;362(9387):900-2.

18. King CL, Malhotra I, Mungai P, Wamachi A, Kioko J, Ouma JH, et al. B cell sensitization to helminthic infection develops in utero in humans. J Immunol. 1998;160(7):3578-84.

19. Fallon PG. Immunopathology of schistosomiasis: a cautionary tale of mice and men. Immunol Today. 2000;21(1):29-35.

20. Chang TH, Smith GW, Riesenman FR, Alston EF. Cerebral granuloma due to schistosomiasis. JAMA. 1948;136:230-8.

21. Roberts M, Cross J, Pohl U, Lucas S, Dean A. Cerebral schistosomiasis. Lancet Infect Dis. 2006;6(12):820.

22. Fowler R, Lee C, Keystone JS. The role of corticosteroids in the treatment of cerebral schistosomiasis caused by Schistosoma mansoni: case report and discussion. Am J Trop Med Hyg. 1999;61(1):47-50. 